myopathy, woolly hair and keratoderma. Hum. Mol. Genet. 9:2761-2766.

4. Rampazzo, A., et al. 2002. Mutation in human desmoplakin domain binding to plakoglobin causes a dominant form of arrhythmogenic right ventricular cardiomyopathy. Am. J. Hum. Genet. 71:1200-1206.

5. Bauce, B., et al. 2005. Clinical profile of four families with arrhythmogenic right ventricular cardiomyopathy caused by dominant desmoplakin mutations. Eur. Heart J. 26:1666-1675.

6. Norman, M., et al. 2005. Novel mutation in desmoplakin causes arrhythmogenic left ventricular cardiomyopathy. Circulation. 112:636-642.

7. Grossmann, K.S., et al. 2004. Requirement of plakophilin 2 for heart morphogenesis and cardiac junction formation. J. Cell Biol. 167:149-160.

8. Gerull, B., et al. 2004. Mutations in the desmosomal protein plakophilin-2 are common in arrhythmogenic right ventricular cardiomyopathy. Nat. Genet. 36:1162-1164.

9. van Tintelen, J.P., et al. 2006. Plakophilin-2 mutations are the major determinant of familial arrhythmogenic right ventricular dysplasia/cardiomyopathy. Circulation. 113:1650-1658.

10. Protonotarios, N., et al. 2001. Genotype-phenotype assessment in autosomal recessive arrhythmogenic right ventricular cardiomyopathy (Naxos disease) caused by a deletion in plakoglobin. J. Am. Coll. Cardiol. 38:1477-1484.

11. Alcalai, R., Metzger, S., Rosenheck, S., Meiner, V. and Chajek-Shaul, T. 2003. A recessive mutation in desmoplakin causes arrhythmogenic right ventricular dysplasia, skin disorder, and woolly hair. J. Am. Coll. Cardiol. 42:319-327.

12. Jamora, C., and Fuchs, E. 2002. Intercellular adhesion, signalling and the cytoskeleton. Nat. Cell Biol. 4:E101-E108

13. Kaplan, S.R., et al. 2004. Remodeling of myocyte gap junctions in arrhythmogenic right ventricular cardiomyopathy due to a deletion in plakoglobin (Naxos disease). Heart Rhythm. 1:3-11.

14. Kostetskii, I., et al. 2005. Induced deletion of the $\mathrm{N}$-cadherin gene in the heart leads to dissolution of the intercalated disc structure. Circ. Res. 96:346-354.

15. Huelsken, J., and Birchmeier, W. 2001. New aspects of Wnt signaling pathways in higher vertebrates. Curr. Opin. Genet. Dev. 11:547-553.

16. Garcia-Gras, E., et al. 2006. Suppression of canonical Wnt $/ \beta$-catenin signaling by nuclear plakoglobin recapitualtes phenotype of arrhythmogenic right ventricular cardiomyopathy. J. Clin. Invest.
116:2012-2021. doi:10.1172/JCI27751

17. Gallicano, G.I., et al. 1998. Desmoplakin is required early in development for assembly of desmosomes and cytoskeletal linkage. J. Cell Biol. 143:2009-2022.

18. Ross, S.E., et al. 2000. Inhibition of adipogenesis by Wnt signaling. Science. 289:950-953.

19. Tzahor, E., and Lassar, A.B. 2001. Wnt signals from the neural tube block ectopic cardiogenesis. Genes Dev. 15:255-260.

20. Marvin, M.J., Di Rocco, G., Gardiner, A., Bush, S.M., and Lassar, A.B. 2001. Inhibition of Wnt activity induces heart formation from posterior mesoderm. Genes Dev. 15:316-327.

21. Schneider, V.A., and Mercola, M. 2001. Wnt antagonism initiates cardiogenesis in Xenopus laevis. Genes Dev. 15:304-315.

22. Lickert, H., et al. 2002. Formation of multiple hearts in mice following deletion of beta-catenin in the embryonic endoderm. Dev. Cell. 3:171-181.

23. Hurlstone, A.F., et al. 2003. The Wnt/beta-catenin pathway regulates cardiac valve formation. Nature. 425:633-637.

24. Pilichou, K., et al. 2006. Mutations in desmoglein-2 gene are associated with arrhythmogenic right ventricular cardiomyopathy. Circulation. 113:1171-1179.

\title{
Neuropeptide signaling and hydrocephalus: SCO with the flow
}

\begin{abstract}
David J. Picketts
Molecular Medicine Program, Ottawa Health Research Institute, and Department of Medicine, Department of Biochemistry, Microbiology, and Immunology, and Centre for Neuromuscular Disease, University of Ottawa, Ottawa, Ontario, Canada.
\end{abstract}

Congenital hydrocephalus affects $0.1-0.3 \%$ of live births, with a high mortality rate $(-50 \%)$ in the absence of surgical intervention. Although the insertion of shunts alleviates the symptoms of the majority of congenital cases, the molecular basis of hydrocephalus and the mechanisms of cerebrospinal fluid (CSF) circulation remain largely unknown. Two important players are the subcommissural organ/Reissner's fiber (SCO/RF) complex and the ventricular ependymal (vel) cells that together facilitate the flow of the CSF through the narrow canals of the ventricular system. In this issue of the JCI, Lang et al. demonstrate that overexpression of the pituitary adenylate cyclase-activating polypeptide (PACAP) type I (PAC1) receptor gene results in abnormal development of the $\mathrm{SCO}$ and vel cells, leading to congenital hydrocephalus (see the related article beginning on page 1924). The ligand for the PAC1 receptor is the neuropeptide PACAP, which uncovers what the authors believe to be a novel role for this signaling cascade in the regulation of CSF circulation.

Hydrocephalus arises from an accumulation of cerebrospinal fluid (CSF), most

Nonstandard abbreviations used: CREB, cAMP response element-binding protein; $\mathrm{CSF}$, cerebrospinal fluid; IFT, intraflagellar transport; PAC1, PACAP type I; PACAP, pituitary adenylate cyclase-activating polypeptide; RF, Reissner's fiber; SCO, subcommissural organ; vel, ventricular ependymal.

Conflict of interest: The author has declared that no conflict of interest exists.

Citation for this article: J. Clin. Invest. 116:1828-1832 (2006). doi:10.1172/JCI29148. frequently due to an impairment of CSF flow within either the ventricular system (noncommunicating hydrocephalus) or the subarachnoid space (communicating hydrocephalus). The CSF is secreted from the choroid plexus, and movement through the ventricular system occurs in a rostrocaudal direction, from the lateral ventricles to the third ventricle via the foramen of Munro, then through the Sylvian aqueduct to the fourth ventricle, and finally into the cisterna magna of the sub- arachnoid space and the central canal of the spinal cord (Figure 1). Ultimately, CSF fluid is removed through the arachnoid villi into the venous circulation.

Noncommunicating hydrocephalus results from an obstruction of the ventricular system and has many causes, including viral infection, tumors, hemorrhage, and developmental defects (1). Obstruction usually occurs in the narrowed segments of the ventricular system, typically the cerebral aqueduct. Indeed, stenosis of the cerebral aqueduct is considered the primary cause of congenital hydrocephalus $(1,2)$. Several factors play a role in the maintenance of CSF flow through the narrow canals, including ciliary movement on ependymal cells and a functioning subcommissural organ (SCO), an ependymal gland located in the dorsocaudal region of the third ventricle at the entrance of the Sylvian aqueduct (Figure 2$)(3,4)$. It is well established that the SCO secretes glycoproteins that aggregate and form a long, threadlike structure known as Reissner's fiber (RF) in most vertebrate species (Figure 2). RF elongates and extends through 


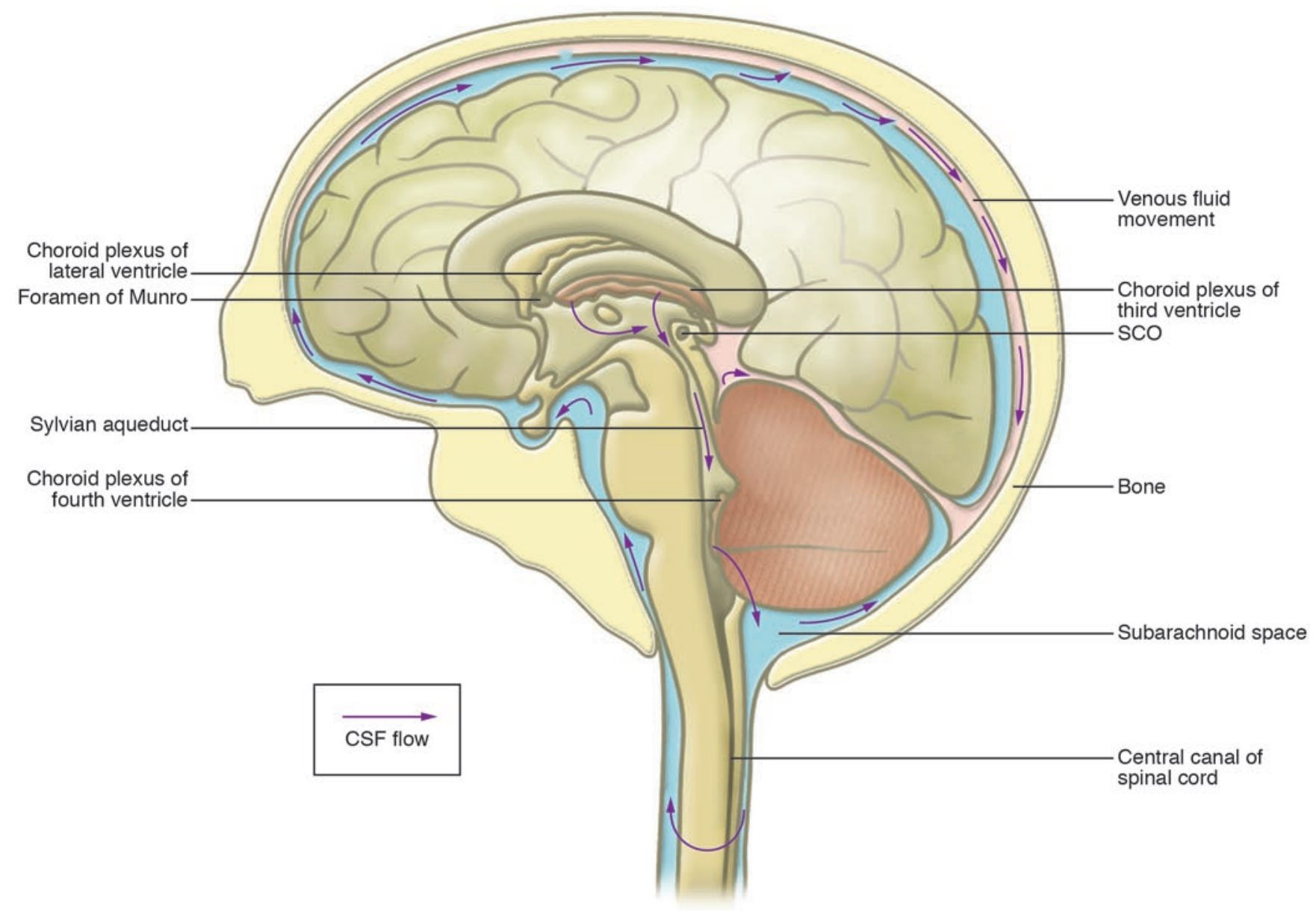

Figure 1

CSF flow and the SCO. CSF is secreted from the choroid plexus and travels through the ventricular system in a rostrocaudal direction from the lateral ventricles to the third ventricle via the foramen of Munro, then through the Sylvian aqueduct to the fourth ventricle, and finally into the cisterna magna of the subarachnoid space and the central canal of the spinal cord. CSF fluid is removed through the arachnoid villi into the venous circulation.

the Sylvian aqueduct to the central canal of the spinal cord and prevents closure of the aqueduct while maintaining CSF flow (4-6). Although such a threadlike fiber has not been found in humans, there is evidence of secreted glycoproteins that remain soluble; these are thought to facilitate flow through the aqueduct (7). Moreover, malformation of the SCO has been identified in several rat and mouse strains that develop spontaneous hydrocephalus (8-10) and in transgenic mouse strains in which the expression of specific genes have been altered (11-14). In this regard, the article by Lang et al. in this issue of the JCI confirms the importance of the SCO in the development of hydrocephalus and implicates the pituitary adenylate cyclase-activating polypeptide (PACAP) neuropeptide signaling cascade in the process (15).

\section{Mice with increased PAC1 receptor expression}

Lang et al. (15) used a bacterial artificial chromosome transgenic approach to generate 3 transgenic mouse lines that contained 2, 4, or 6 additional copies of the PACAP type I (PAC1) receptor gene. The expression of this transgene recapitulated normal PAC1 expression in the CNS and was accompanied by a reduction in the cerebral cortex and corpus callosum as well as dose-dependent features of hydrocephalus, including enlarged lateral and third ventricles and a reduced SCO, that resulted in increased death in the early postnatal period. It is not surprising that these animals present with a complex phenotype given the vast number of functions attributed to the neuropeptide PACAP, which is the main ligand for the PAC1 receptor (16). In the CNS alone, PACAP has been shown to act as a neuromodulator, neurotransmitter, neurotrophic factor, and neuroprotective factor $(16,17)$. Moreover, it is unclear whether overexpression of the PAC1 receptor leads to increased activity from increased binding of its ligand (i.e., PACAP is not limiting) or by responding to other ligands with which it would not normally interact (i.e., PACAP is limiting). Indeed, teasing apart the various biological activities of PACAP/PAC1 receptor function to explain the observed phenotype in this model poses a challenge. Nonetheless, Lang et al. have done a remarkable job at characterizing the molecular basis for the hydrocephalus in these mice.

\section{PAC1 receptor and the development of the SCO}

A common feature in animal models of hydrocephalus is the lack of SCO or an 

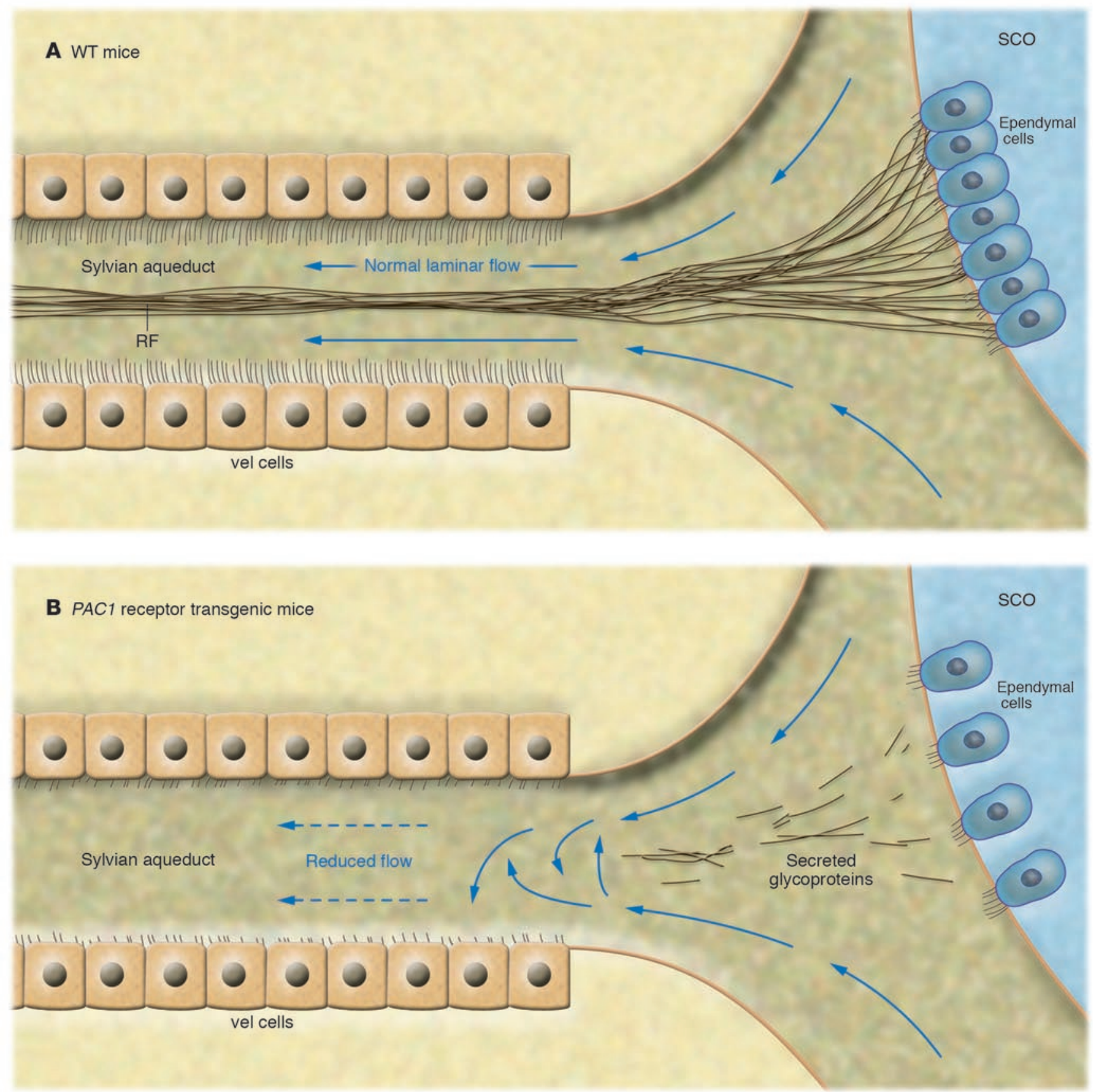

\section{Figure 2}

Schematic diagram modeling flow through the Sylvian aqueduct in WT and PAC1 receptor transgenic mice. (A) In WT mice, ependymal cells from the SCO secrete glycoproteins that form RF and help maintain laminar flow of the CSF through the aqueduct. Cilia on the vel cells also promote CSF flow. (B) In transgenic animals, there are fewer SCO cells, which may result in absent or disorganized RF but not stenosis of the aqueduct. In addition, shorter, inefficient cilia are present on the vel cells. Together, these defects likely result in turbulent CSF flow at the entrance (curved arrows) and reduced flow within (dashed arrows) the cerebral aqueduct, thereby promoting hydrocephalus.

abnormally developed SCO (reviewed in refs. 1, 3). Such an observation was also made by Lang et al. (15); the size and cell number of the SCO were dramatically reduced through enhanced apoptosis. Mouse and rat mutants that spontaneous- ly develop hydrocephalus and present with malformed SCO often develop stenosis of the aqueduct (1). However, the PAC1 transgenic mice showed an enlarged aqueduct, suggesting that the underlying mechanism may be different (see Figure 4 in ref. 15).
As the PAC1 receptor is normally expressed in the cells of the SCO, it implies that PACAP signaling may normally regulate the secretion of glycoproteins into the CSF through PKA or PKC signaling conduits (Figure 3). Overproduction of 


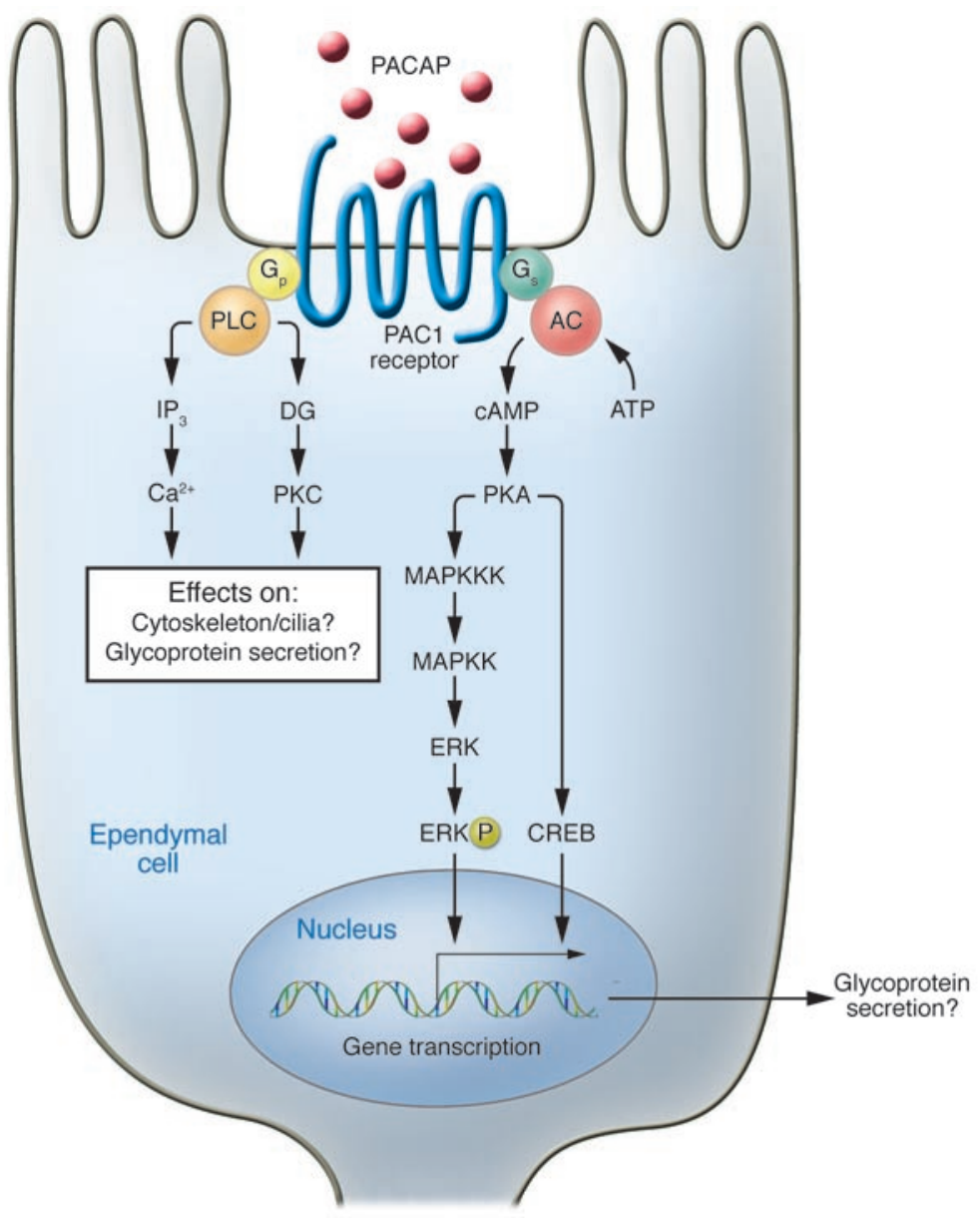

secreted glycoproteins may lead to aggregate accumulation at the entrance of the aqueduct that could result in turbulent or reduced flow of the CSF through the canal. Consistent with such a mechanism is the finding that PACAP signaling stimulates insulin secretion (18). Moreover, analysis of the SCO in the hyh mouse mutant showed an increase in secretory activity (8). Alternatively, if PACAP signaling inhibited glycoprotein secretion, it may interfere with the correct formation of RF and with CSF flow. This latter possibility seems less likely, since the absence of the RF often leads to stenosis of the aqueduct, which was not observed. A third possibility is that the atrophic SCO leads to deficiencies in the RF such that it cannot properly regulate the monoamine concentration in the CSF. Increased levels of monoamines could feedback to the choroidal cells of the choroid plexus, resulting in increased CSF production. Additional experiments are required to determine the normal effect of PACAP signaling on the function of the ependymal cells of the SCO and the subsequent formation of the RF.

\section{CSF flow via ciliary movement}

PAC1 receptor overexpression in the ventricular ependymal (vel) cells also contributes significantly to the hydrocephalus in this model (15). The ciliary beating of the vel cells is important for laminar flow of CSF in the narrow canals, as best demonstrated by primary ciliary dyskinesia, a syndrome that impairs ciliary activity and results in hydrocephalus $(19,20)$. Lang et al. demonstrated that the vel cells of the PAC1 transgenic mice had increased phosphorylated cAMP response element-binding protein (CREB) activity and fewer, disorganized cilia as shown by tubulin staining. Another component of the intraflagellar transport (IFT) complex, Polaris, was normal, as were the choroidal ependymal cells, which do not express significant levels of PAC1 receptor. It is unclear how increased PACAP signaling may impinge on the IFT complex or ciliary activity, but it most likely represents a gain-of-function effect through increased PKA or PKC signaling (Figure 3 ). In this regard, the authors demonstrated increased phosphorylation of myristoylated, ala-

\section{Figure 3}

Potential mechanisms of increased PAC1 receptor signaling. Schematic representation of PACAP/PAC1 receptor signaling through the PKA and PKC pathways. AC, adenylyl cyclase; DG, diacylglycerol; $\mathrm{IP}_{3}$, inositol triphosphate; MAPKK, MAPK kinase; MAPKKK, MAPKK kinase. nine-rich C-kinase substrate (MARCKS), a cytoskeleton-associated factor, albeit in a whole-brain extract.

\section{Summary and future directions}

The study by Lang et al. (15) represents what I believe to be a novel role for the PACAP neuropeptide signaling pathway. It is likely that a combination of reduced cell number within the SCO, disrupted function of the SCO (aberrant RF), and abnormal cilia function of the vel cells lining the cerebral aqueduct significantly alter the laminar flow through this narrow channel and initiate or promote the development of hydrocephalus (Figure 2). The challenge will be to define the precise role of the PAC1 receptor and PACAP signaling in SCO development and the coordination of CSF flow.

Address correspondence to: David Picketts, Ottawa Health Research Institute, 501 Smyth Road, Ottawa, Ontario K1H8L6, Canada. Phone: (613) 737-8989; Fax: (613) 737-8803; E-mail: dpicketts@ohri.ca. 
1. Perez-Figares, J.M., Jimenez, A.J., and Rodriguez, E.M. 2001. Subcommissural organ, cerebrospinal fluid circulation, and hydrocephalus. Microsc. Res. Tech. 52:591-607.

2. Rizvi, R., and Anjum, Q. 2005. Hydrocephalus in children. J. Pak. Med. Assoc. 55:502-507.

3. Galarza, M. 2002. Evidence of the subcommissural organ in humans and its association with hydrocephalus. Neurosurg. Rev. 25:205-215.

4. Rodriguez, E.M., Rodriguez, S., and Hein, S. 1998. The subcommissural organ. Microsc. Res. Tech. 41:98-123.

5. Vio, K., et al. 2000. Hydrocephalus induced by immunological blockage of the subcommissural organ-Reissner's fiber (RF) complex by maternal transfer of anti-RF antibodies. Exp. Brain Res. 135:41-52.

6. Grondona, J.M., et al. 1998. Neuraminidase injected into the cerebrospinal fluid impairs the assembly of the glycoproteins secreted by the subcommissural organ preventing the formation of Reissner's fiber. Histochem. Cell Biol. 109:391-398.

7. Rodriguez, E.M., Garrido, O., and Oksche, A. 1990 Lectin histochemistry of the human fetal subcommissural organ. Cell Tissue Res. 262:105-113.

8. Perez-Figares, J.M., et al. 1998. Spontaneous congenital hydrocephalus in the mutant mouse hyh.
Changes in the ventricular system and the subcommissural organ. J. Neuropathol. Exp. Neurol. 57:188-202.

9. Takeuchi, I.K., Kimura, R., Matsuda, M., and Shoji, R. 1987. Absence of subcommissural organ in the cerebral aqueduct of congenital hydrocephalus spontaneously occurring in MT/HokIdr mice. Acta Neuropathol. (Berl.). 73:320-322.

10. Takeuchi, I.K., Kimura, R., and Shoji, R. 1988. Dysplasia of subcommissural organ in congenital hydrocephalus spontaneously occurring in CWS/ Idr rats. Experientia. 44:338-340.

11. Estivill-Torrus, G., Vitalis, T., Fernandez-Llebrez, P. and Price, D.J. 2001. The transcription factor Pax6 is required for development of the diencephalic dorsal midline secretory radial glia that form the subcommissural organ. Mech. Dev. 109:215-224.

12. Fernandez-Llebrez, P., et al. 2004. Msx1-deficient mice fail to form prosomere 1 derivatives, subcommissural organ, and posterior commissure and develop hydrocephalus. J. Neuropathol. Exp. Neurol. 63:574-586.

13. Krebs, D.L., et al. 2004. Development of hydrocephalus in mice lacking SOCS7. Proc. Natl. Acad. Sci.U.S. A. 101:15446-15451.

14. Louvi, A., and Wassef, M. 2000. Ectopic engrailed 1 expression in the dorsal midline causes cell death, abnormal differentiation of circumventricular organs and errors in axonal pathfinding. Development. 127:4061-4071.

15. Lang, B., et al. 2006. Expression of the human PAC1 receptor leads to dose-dependent hydrocephalus-related abnormalities in mice. J. Clin. Invest. 116:1924-1934. doi:10.1172/JCI27597.

16. Vaudry, D., et al. 2000. Pituitary adenylate cyclaseactivating polypeptide and its receptors: from structure to functions. Pharmacol. Rev. 52:269-324.

17. Zhou, C.J., et al. 2002. PACAP and its receptors exert pleiotropic effects in the nervous system by activating multiple signaling pathways. Curr. Protein Pept. Sci. 3:423-439.

18. Filipsson, K., Kvist-Reimer, M., and Ahren, B. 2001. The neuropeptide pituitary adenylate cyclase-activating polypeptide and islet function. Diabetes. 50:1959-1969.

19. Greenstone, M.A., Jones, R.W., Dewar, A., Neville, B.G., and Cole, P.J. 1984. Hydrocephalus and primary ciliary dyskinesia. Arch. Dis. Child. 59:481-482.

20. Nakamura, Y and Sato, K. 1993. Role of disturbance of ependymal ciliary movement in development of hydrocephalus in rats. Childs Nerv. Syst. 9:65-71.

\title{
Liver $\mathrm{X}$ receptor opens a new gateway to StAR and to steroid hormones
}

\author{
Colin R. Jefcoate
}

Department of Pharmacology, University of Wisconsin Medical School, Madison, Wisconsin, USA.

\begin{abstract}
Liver X receptors (LXRs) broadly limit cholesterol accumulation by regulating expression of genes involved in cholesterol efflux and storage. In this issue of the JCI, Cummins et al. report that LXR $\alpha$ is involved in similar regulation in the adrenal cortex, but it also substantially modulates glucocorticoid synthesis (see the related article beginning on page 1902). LXR $\alpha$ deletion in mice increases the availability of adrenal cholesterol for steroid synthesis by decreasing the expression of cholesterol efflux transporters. Glucocorticoid synthesis requires intramitochondrial cholesterol transport mediated by the steroidogenic acute regulatory protein (StAR). Surprisingly, LXR deletion and stimulation by an agonist each increase glucocorticoid synthesis. This parallels increased expression of StAR and several other steroidogenic genes.
\end{abstract}

\section{Liver $\mathrm{X}$ receptors stimulate expression of genes that lower cholesterol}

Liver X receptors (LXRs) belong to a class of ligand-dependent nuclear receptors

Nonstandard abbreviations used: ABCA1, $\mathrm{ABC}$ transporter A1; CYP11A1, cytochrome

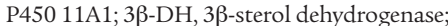
FAS, fatty acid synthase; HSL, hormone-sensitive lipase; LXR, liver X receptor; RXR, retinoid X receptor; SR-B1, scavenger receptor-B1; StAR, steroidogenic acute regulatory protein; $\mathrm{T} 1317$, LXR agonist T090137.

Conflict of interest: The author has declared that no conflict of interest exists.

Citation for this article: J. Clin. Invest. 116:1832-1835 (2006). doi:10.1172/JCI29160. that includes the PPAR family and receptors for vitamin $\mathrm{D}$, thyroid hormone, and retinoic acid, which form heterodimers with retinoid $X$ receptors (RXRs). In doing so, LXRs exert transcriptional control on cholesterol and fatty acid homeostasis in a variety of cell types. Two forms of the LXR have been identified: LXR $\alpha$ is expressed at high levels in liver but also at more modest levels in cells that are involved in cholesterol transport and metabolism (e.g., intestines, adipose, macrophages, kidney, and lung) while $\operatorname{LXR} \beta$ is broadly expressed. LXRs are known to control the expression of genes involved in the transport of excess cholesterol from peripheral tissues to the liver (e.g., the $\mathrm{ABC}$ family of membrane transporters, including ABC transporter A1 [ABCA1], ABCG5, ABCG8, and ABCG1), as well as hepatic metabolism of this cholesterol by cytochrome P450 7A1 (CYP7A1) to bile acids (1). The roles of LXRs have recently been expanded through the study of mice deficient in LXR $\alpha$ and LXR $\beta$ as well as the use of the LXR agonist T090137 (T1317), which stimulates both receptors (1). Mice deficient in LXR $\alpha$, LXR $\beta$, or both receptors are fully functional, which indicates that the cholesterol transport processes and fatty acid changes regulated by these receptors play a modulating rather than essential role. The link between the LXRs and cholesterol homeostasis was firmly established by the finding that mice deficient in LXR $\alpha$ lose the ability to convert cholesterol to bile acids in the liver and as a result accumulate cholesterol esters in their liver when challenged with a cholesterol-rich diet (1). LXRs also have extensive extrahepatic functions, including regulation of fatty acid and cholesterol metabolism in macrophages - a critical process in the inflammatory response 